\title{
Geología del subsuelo del Área Metropolitana del Gran Resistencia (AMGR), Provincia del Chaco, Nordeste de Argentina
}

ROBERTO TORRA

\section{Resumo}

O presente trabalho apresenta os resultados de um estudo geológico e geotécnico básico feito em sedimentos inconsolávels limosos e argilosos que constituem o solo e subsolo do Area Metropolitana del Gran Resistencia (AMGR) e zonas limítrofes. Fizeram-se estudos básicos em sedimentologia e geotecnia com o objeto de relacionar suas caraterísticas em textura e composição, alem disso, verificar sua interrelação e comportamento. Os ensaios geotécnicos específicos de mecânica do solo feitos forom: límite líquido, límite plástico, índice plástico e granulometria. $\mathrm{O}$ area de estudo esta sempre caraterizada por uma sucessão inconsolidável, composta de camadas horizontais de limos finos ou grossos (a areia muito fina representa sempre percentagens inferiores ao $10 \%$ ) ligação a camadas argilosas maciças apaixonadamente edafizadas e regolitizadas seja que os sedimentos se encontrarem como afloramentos ou a pouca profundidade (0 $10 \mathrm{~m}$ ). Essas camadas correspondem a Formação Pampeana sensu lato de idade Pleistocena-Holoceno. Mais por embaixo dessas camadas meteorizadas ou regolitizadas encontra-se uma monótona sucessão heterolitica composta por arenitos supermaduras de cor branco bem arredondadas e bem selecionadas com intercalações de camadas de argila maciça cor castanho averdengado, predominantemente montmorilloníticas. A zona de contato pode ser definivel 
como espalhado e transicional, sendo a macro e meso arquitetura completamente horizontal. Esta sucessão de camadas areno-argilosas corresponde à Formação Ituzaingó de idade Mioceno Média. A mesma apresenta excelentes afloramentos nos barrancos da imagem à esquerda do Rio Paraná com iguais características sedimentológicas e geotécnicas só $2 \mathrm{~km}$ de distancia da area de investigação. Os afloramentos dessa formação encontram-se ao longo da imagem esquerda do Rio Paraná na Províncias de Corrientes e Entre Rios. Os mesmos são o resultado do fraturamento extensional da idade Quaternária. O estudo analisa os resultados dos ensaios mecânicos e sua relação com as litofácies sedimentógicas reconhecidas, mesmo asim, relaciona-se tambem com os verificaçãos encontrados em datos de perfurações hidrogeológicas disponivels na zona de trabalho. A grande quantidade de problemas de assentamentos ou afundamentos que caracterizan o area de estudo podem estar relacionados com a grande erodabilidade dos materiais argilosos expandivels presentes nos sedimentos da Formação Pampeana.

Palavras Chave: Geologia, Geotecnia, Correlações de litofácies, Formaçãos Pampeana e Ituzaingó, Área Metropolitana Gran Resistencia (AMGR), Nordeste de Argentina

\section{Resumen}

El presente trabajo presenta los resultados de un estudio geológico realizado en sedimentos inconsolidados limosos, limo-arenosos y arcillosos que constituyen el suelo y subsuelo poco profundo del Area Metropolitana del Gran Resistencia (AMGR) y zonas aledañas. Se llevaron a cabo estudios estándares y básicos de sedimentología y geotecnia con el objetivo de caracterizar sus propiedades texturales y composicionales como así también determinar su interrelación y comportamiento geométrico. Los ensayos geotécnicas específicos de mecánica de suelo realizados fueron: límite líquido, límite plástico, índice plástico y granulometria. La zona de estudio se caracteriza por una sucesión inconsolidada de capas horizontales y conformes de arcillas y/o capas limosas y limo-arenosas (con menos de $10 \%$ de arena muy fina) parcialmente edafizadas y/o regolitizadas. Esta situación se repite tanto en los materiales sedimentarios que yacen como afloramientos o a muy poca profundidad. Estas capas superiores ( 0 a $15 \mathrm{~m}$ de profundidad) corresponden a la Formación Pampeana sensu lato de edad Pleistoceno-Holoceno. Por debajo de estas capas meteorizadas y/o regolitizadas se dispone de manera transicional, y también con 
geometría horizontal, una monótona sucesión heterolítica compuesta por areniscas supermaduras blanquecinas bien redondeadas y bien seleccionadas con intercalaciones de capas de arcillas macizas castaño-verdoso. Esta sucesión de capas areno-arcillosas constituyen la Formación Ituzaingó. Su edad corresponde al Mioceno Medio. La misma presenta excelentes afloramientos en las barrancas de la margen izquierda del Río Paraná con iguales características sedimentológicas y geotécnicas a tan sólo $2 \mathrm{~km}$ de distancia del área investigada, sobre la margen izquierda del Río Paraná en la Provincia de Corrientes. Estos afloramientos son el resultado de un fracturamiento extensional de edad Cuaternaria. El estudio compara los resultados de los ensayos mecánicos con las litofacies sedimentológicas reconocidas como así también se integra con otros datos encontrados en los registros de perforaciones hidrogeológicas disponibles en la zona de trabajo. La gran cantidad de problemas de asentamientos y/o hundimientos, presentes en ésta zona, estarían relacionados con la elevada erodabilidad, lavado y asentamiento de los materiales arcillosos constituyentes de los sedimentos superiores de la Formación Pampeana.

\section{Introducción}

El Area Metropolitana del Gran Resistencia (AMGR) se encuentra localizada en la planicie fluvial del Río Paraná sobre su margen derecha (Figura 1). Asimismo una parte del conurbano se encuentra sobrepasando el límite o escarpa de la terraza fluvial del valle del Río Paraná (MAURO et al, 1998; TORRA, 1994). Esta área de importancia geoestratégica posee a la fecha escasos estudios de investigación básica geológica del subsuelo poco profundo $(0,00$ 100,00 mbbp).

Sin embargo, hay que destacar que existe un gran número de estudios técnicos regionales generales sobre los suelos sólo superficiales de tipo arcillosos e hidromórficos presentes en esta comarca y que fueran realizados en sucesivas etapas por profesionales del PEA (Programa Estratégico de Acción para la Cuenca del Bermejo). Estos estudios fueron plasmados en una sucesión de informes técnicos de carácter inédito hasta la fecha (PEA EI. 1.1. 1999a; PEA EI. 2.5. 1999b; PEA EI. 1.1. 1999c). Asimismo, el PEA también elaboró varios informes inéditos relacionados con el potencial social y humano de la región, cartografía multitemática medioambiental y cartografía de suelos entre los que podemos destacar los siguientes: PEA El. 2.6., 1999d; PEA El. 6.1., 1999e; PEA El. 6.1., 1999f. Estos 
informes no se refieren específicamente a los aspectos geológicos ni ingenieriles (mecánica de suelo) de la región. El estilo de relieve que presenta el área de estudio está caracterizado por una amplia llanura de inundación (30 km de ancho) desarrollada en el valle fluvial del Río Paraná, topográficamente plana y con escasas pendientes.

En este trabajo solamente nos referiremos a las características geológicas-sedimentológicas y algunos parámetros básicos de mecánica de suelos de los materiales presentes en el subsuelo poco profundo correspondiente al Area Metropolitana del Gran Resistencia (AMGR) y sus alrededores (Figuras 1 y 7 ).

Uno de los principales objetivos de la presente contribución ha sido correlacionar las características geotécnicas más significativas de los resultados encontrados en ensayos de suelos realizados a partir de muestras coleccionadas en destapes artificiales en las capas de sedimentos del Cuaternario y/o Terciario superior sensu lato.

Se analiza la posibilidad de realizar correlaciones en las distintas capas de sedimentos en los $-15 \mathrm{mbbp}$ de profundidad hecho que resulta siempre factible en caso de disponer de un número significativo de perforaciones regularmente distribuidas. A pesar del reducido número de perforaciones estudiadas (ocho perforaciones en total) se logró una buena precisión en la correlación de litofacies. Este hecho fue factible mediante el empleo de las propiedades texturales, composicionales, tonales y mecánicas lo que está fundamentalmente basado en su notable similitud tanto textural como composicional y estructural que presentan las litofacies a lo largo de grandes extensiones en esta región. Por debajo de ésta profundidad (-15 mbbp) se encuentra una sucesión heterolítica monótona en cuanto a su composición litológica caracterizada por la presencia de litofacies horizontales psamíticas (arenisca supermadura fina blanquecina muy bien redondeada, a veces amarillenta por impregnaciones limoníticas) y capas de pelitas macizas castaño verdoso. Esta secuencia, en cierto modo escapa al alcance de las fundaciones de ingeniería civil rutinarias (edificios de pequeño a mediano porte, fundaciones para algunas torres de transmisión eléctrica, canales, puentes, alcantarillas, algunos ductos, etc.).

\section{Metodología}

Los métodos empleados fueron el estudio de registros de perforaciones, análisis y mediciones de laboratorio y observaciones de detalle en cortes artificiales próximos a la ciudad de Resistencia 
con levantamiento de perfiles sedimentológicos. También contribuyeron a realizar éste artículo el estudio y análisis de bibliografía así como el conocimiento previo del autor sobre la geología y geomorfología de la comarca.

Para el estudio previsto en el presente trabajo se coleccionaron siete muestras seleccionadas a saber:

1) En los destapes artificiales próximos a la Cerámica Toba se coleccionaron las muestras HI-HS-CH-01-98 y HI-HI-CH-02-98 (Figuras 4 y 5$)$.

2) En un destape artificial en el centro de la ciudad de Resistencia se tomaron las muestras RE-PYP-CH-01-99 y TI-ELF-CH-01-99.

3) Finalmente, en el corte artificial originado a partir de la construcción del Canal Derivador del Río Negro hacia el Río Salado se coleccionaron las muestras CD-LB-CH-01-98, CD-LB-CH-02-98 y CD-LB-CH-03-98 (Figuras 1, 6 y 7 ).

La metodología para la clasificación mecánica de las muestras empleada en laboratorio fue la clásica que figura en la bibliografía mundial de acuerdo con JUÁREZ BADILLO y RICO RODRÍGUEZ, 1982; LAMBE y WHITMANN, 1976; TERZAGUI y PECK, 1980. Las muestras se prepararon convenientemente para los ensayos y los resultados finales fueron volcados de acuerdo con las tablas de clasificación de suelos del HRB ("Highway Research Board") y SUCS (Sistema Unificado de Clasificación de Suelos). En todos los casos fue necesario la obtención de los parámetros de plasticidad: límite líquido, límite plástico, índice de plasticidad, curva granulométrica y lavado sobre tamiz $N^{\circ} 200$ de cada una de las muestras, siguiendo procedimientos normalizados.

Los registros de perforaciones estudiados fueron los denominados como Perforación Resistencia $N^{\circ} 1$ y Perforación Resistencia $N^{\circ} 2$, ambas ejecutadas en 1906 por personal especializado del ex Ferrocarril Francés. La Perforación Escuela Benítez $\mathrm{N}^{\circ} 1$; las Perforaciones Fontana $\mathrm{N}^{\circ} 1$, Fontana $\mathrm{N}^{\circ} 2$ y Fontana $\mathrm{N}^{\circ} 3$; la Perforación Colonia Baranda y la Perforación Puerto Tirol (véase ubicación en la Figura 1).

\section{Resultados}

El análisis de los ocho registros de perforaciones estudiados mostraron en forma consistente e inequivoca un sector superior en la columna estratigráfica, compuesto mayormente por materiales limo-arcillosos, con escasa participación de arena fina y/ o arena muy fina, hasta una profundidad promedio de $-15 \mathrm{mbbp}$. A 
partir de ésta profundidad se pudo reconocer una sucesión compuesta por capas que se vuelven predominantemente arenosas, correspondiendo a una arenisca media, fina o muy fina supermadura, generalmente blanquecina y/o ligeramente amarillenta, con intercalaciones de capas de arcillas macizas castaño verdoso hasta el final de la perforación más profunda (Figuras 2 y 3).

El análisis realizado sobre las siete muestras coleccionadas para el estudio de parámetros mecánicos también mostró resultados consistentes e inequívocos. Los materiales de granulometría fina (limo-arcilla) de color castaño claro siempre resultan ser suelos de tipo CL ("clay low") mientras que los materiales de granulometría fina, predominando la arcilla, resultan ser suelos de tipo $\mathrm{CH}$ ("clay high"). Los materiales clasificados en los perfiles como SM ("sand-silt medium") corresponden al sector intermedio entre las areniscas supermaduras sensu stricto y los materiales suprayacentes (Figuras 2 y 3 ).

En la Tabla 1 se pueden observar los parámetros encontrados para las muestras estudiadas que denotan un claro predominio de suelos de tipo $\mathrm{CL}$.

\begin{tabular}{c|c|c|c|c|c|c}
\hline \multicolumn{1}{c|}{ Muestra } & $\begin{array}{c}\text { Limite } \\
\text { Liquido }\end{array}$ & $\begin{array}{c}\text { Límite } \\
\text { Plástico }\end{array}$ & $\begin{array}{c}\text { indice } \\
\text { Plástico }\end{array}$ & $\begin{array}{c}\text { Tamiz No } \\
200\end{array}$ & S.U.C.S. & H.R.B. \\
\hline HI-HS-CH-01-98 & 53,6 & 20,5 & 33,1 & 86,6 & CH & A-7-6(31) \\
\hline HI-HI-CH-02-98 & 54,2 & 20,8 & 33,4 & 92,2 & CH-MH & A-7-6(33) \\
\hline TI-ELF-CH-01-99 & 31,2 & 17,3 & 13,9 & 75,6 & CL & A-6(8) \\
\hline RE-PYP-CH-01-99 & 34,1 & 17,8 & 16,3 & 93,1 & CL & A-6(15) \\
\hline CD-LB-CH-98-01 & 47,7 & 18,8 & 28,9 & 92,3 & CL & A-7-6(27) \\
\hline CD-LB-CH-98-02 & 47 & 21,1 & 25,9 & 92,1 & CL & A-7-6(24) \\
\hline CD-LB-CH-98-03 & 46,4 & 18,1 & 28,3 & 91,6 & CL & A-7-6(26) \\
\hline
\end{tabular}

Tabla 1. Límite Líquido, Límite Plástico y Clasificación

\section{Interpretación}

La Formación Pampeana ha sido definida como un conjunto de capas de sedimentos limo-arcillosos, predominando los limos gruesos y/o finos (FRENGUELLI, 1945) desde los tiempos de Florentimo Ameghino con sucesivas actualizaciones de otros inves- 
tigadores (AMEGHINO, 1981; CARDOSO, 1911; CORTELEZZI y LERMANN, 1969; FRENGUELLI, 1950; FRENGUELLI, 1955).

En el presente estudio se encuentra que los sedimentos superficiales y/o poco profundos, hasta una profundidad promedio de $-15 \mathrm{mbb}$, corresponden a litofacies sedimentológicas del tipo franco limosa $(\mathrm{CL})$ más litofacies arcillosas subordinadas $(\mathrm{CH})$, con escasa participación de fracción arena muy fina, siempre en proporción inferior a $10 \%$ de roca total.

A mayor profundidad de $-15 \mathrm{mbbp}$, en promedio, se desarrolla una secuencia de estratos atribuibles a una sucesión heterolítica compuesta por capas de areniscas supermaduras finas blanquecinas con algunas delgadas intercalaciones de arcillas macizas castaño verdoso (Figuras 2 y 3 ). Esta sucesión de capas corresponden a los estratos de las Formación Ituzaingó de acuerdo con recientes estudios sedimentológicos (TORRA, 1997; TORRA, 1998a,b,c,d; TORRA, 1999; TORRA y MIÑO, 1999).

Se esboza una correlación de los materiales estudiados desde los puntos de vista sedimentológico y mecánico de la manera en que fueron interpretados en el presente trabajo (Tabla 2).

\begin{tabular}{l|c|c}
\hline \multicolumn{1}{c|}{ Proporciones de materiales } & Sedimentología & Mecánica de suelos (HRB) \\
\hline arcilla $>>$ limo grueso y/o fino & litofacies de arcilla maciza & suelo CH \\
\hline limo $>>>$ arcilla $>>$ arenisca muy fina & litofacies de limolita maciza & suelo CL \\
\hline arenisca $>$ limo $>$ arcilla & litofacies de areno limosa & suelo SM \\
\hline arenisca fina y/o muy fina supermadura blanquecina & litofacies de arenisca supermadura & suelo SM (extrapolado) \\
\hline arcilla maciza castaño verdoso & litofacies de arcilla maciza & suelo CL (extrapolado) \\
\hline
\end{tabular}

Tabla 2. Materiales y litofacies definidas

Los materiales estudiados presentan correlación horizontal como puede apreciarse en las Figuras 2, 3, 6 y 7 lo que descartaría una influencia significativa de procesos geomórficos del sistema fluvial del Río Paraná (TORRA, 2001). Asimismo, la escasa presencia de arena fina o muy fina en disposición horizontal, regular y sin truncaciones resulta otro elemento destacable que también avala la idea de la poca o nula participación fluvial canalizada. La mayor participación debió haber sido de tipo laminar, causante de la meteorización y/o regolitización de las capas superiores. 
De acuerdo con SAYAGO (1999), las características de estos depósitos corresponderían a aquellos de características lagunares y lagunares hidro-eólico, es decir sedimentos acumulados en zonas con ligera depresión de relieve en los cuales el aporte fluvial y/o eólico fue el proceso predominante. Asimismo, las secciones superiores han sufrido un proceso de retrabajamiento por acción fluvial (paleolagunas) y/o pluvial lo que puede conllevar a un importante enriquecimiento en la fracción limo por lavado de arcillas erodibles (PYE, 1995).

Los procesos mencionados ocurrieron especialmente en la parte superior del Pleistoceno y Holoceno (TORRA, 1994; TORRA, 2001), período en el cual se produjeron oscilaciones climáticas entre las que hay que destacar etapas más húmedas con su consecuente aumento en las precipitaciones pluviales (IRIONDO, 1999).

A partir del estudio de los registros de perforaciones y otros estudios anteriores sobre el subsuelo y paleogeografía de la región (TORRA, 1994; TORRA, 1998b), se elaboró la Figura 8 en la cual puede observarse, de forma simplificada, la estructura geológica del valle fluvial del Río Paraná a la altura del AMGR. En esta Figura puede observarse un fracturamiento extensional típico que controla el valle fluvial del Río Paraná desde Corrientes hasta Rosario aproximadamente (TORRA, 2002a).

\section{Conclusiones}

En base a los resultados encontrados se pueden expresar las siguientes conclusiones relacionadas con las capas de sedimentos que integran el subsuelo poco profundo del área estudiada:

Presencia de suelos de tipo CL (franco limoso) hasta aproximadamente $-15 \mathrm{mbbp}$.

Presencia de suelos de tipo $\mathrm{CH}$ (arcillo-limoso) hasta aproximadamente $-15 \mathrm{mbbp}$ intercalados en los suelos $\mathrm{CL}$ (francolimoso).

Un espesor promedio de aproximadamente 15 metros para el cuerpo sedimentario correspondiente a la Formación Pampeana. Geometría de disposición horizontal y conforme sin ninguna presencia de discordancia de erosión ni otra clase de truncamientos.

Contacto transicional largo $(2-5 \mathrm{~m})$ con las unidades infrayacentes mediante capas arcillo-arenosas inconsolidadas de tipo 
SM (capas areno-limosas).

Areniscas supermaduras blanquecinas (areniscas de alta densidad), con intercalaciones de arcillas macizas castaño verdoso que corresponden a litofacies marinas de mar abierto y/o facies litorales de la Formación Ituzaingó (TORRA, 1998a; TORRA, 2001; TORRA, 2002a).

Los suelos y/o materiales inconsolidados de tipo CL y $\mathrm{CH}$ presentes en la Formación Pampeana son considerados correlacionables con las Formaciones La Plata (suelos $C L$ ) y Luján (suelos CH) (CORTELEZZI y LERMANN, 1969) respectivamente. Las Formaciones La Plata y Luján que se encuentran definidas en la Provincia de Buenos Aires son correlacionadas aquí con los sedimentos de la Formación Pampeana (suelos $\mathrm{CL}$ y $\mathrm{CH}$ de la sección superior de la columna). Esto se debe a su inequívoca igualdad textural, estructural y composicional como así también al marco geológico y geomorfológico regional (TORRA, 2001; TORRA, 2002b). Asimismo, estas formaciones presentan un perfil geoquímico de elementos trazas completamente similar (TORRA, 2000; TORRA, 2002b).

En lo relacionado con las tareas de fundaciones para obras civiles (diques, represas, puentes, terraplenes, alcantarillas), especial atención debería tenerse en cuenta al momento de las decisiones estratégicas, como por ejemplo si las mismas quedarán empotradas en suelos de tipo CL o atravesarán largas secciones de éste tipo de material. Este hecho se vincula con la significativamente alta posibilidad de lavado y erodabilidad de arcillas expansibles en dichos suelos lo que ocasionaría posteriores problemas en las estructuras ingenieriles tales como asentamientos y hundimientos lo que produciría el colapso de las estructuras. Este fenómeno de erodabilidad y posterior asentamiento debería ser analizado cuidadosamente en la etapa previa a la realización de proyectos de obras civiles de mediana o grandes dimensiones.

Finalmente resultaría de sumo interés estudiar con mayor detalle las capas dispuestas por debajo de los $-15 \mathrm{mbbp}$ debido a que un exacto conocimiento de la sucesión psamítica-pelítica sería de significativa importancia en la estimación del cálculo preliminar de la profundidad del pilotaje en obras de ingeniería de mediano o gran porte. Podemos citar como ejemplo la fundación de represas de gravedad de hormigón armado. También puede ser citado el segundo puente ferro-vial en etapa de ante-proyecto entre las ciudades de Corrientes y Resistencia y otros tres probables sitios de emplazamiento de viaductos con similares características (ante- 
proyectos), localizados sobre el valle fluvial del Río Paraná un poco más al sur del área estudiada $(300 \mathrm{~km})$ entre las Provincias de Corrientes y Santa Fé. Las condiciones geológicas y geomorfológicas de estas zonas son completamente iguales a las presentes en el área estudiada por lo cual este trabajo podría servir como un estudio piloto básico. 


\section{Trabajos citados en el texto}

AMEGHINO, F. La Formación Pampeana o estudio de los terrenos de transporte de la cuenca del Plata. Buenos Aires-Paris. 371 p., 1881.

CARDOSO, A. Buenos Aires en 1516. Anales del Museo Nacional de Buenos Aires. XXI. Buenos Aires. pp. 309-372, 1911.

CORTELEZZI, C., R. y LERMANN, J. C. Estudio de las Formaciones Marinas de la Costa Atlántica de la Provincia de Buenos Aires. VIII Congreso Mundial del INQUA. Paris. pp. 135-164 (reimpresión del LEMIT, La Plata), 1969.

FRENGUELLI, J. El Piso Platense. Revista del Museo de La Plata. Geología. II. 287-311. La Plata, 1945.

FRENGUELLI, J. Rasgos generales de la morfología y la geología de la Provincia de Buenos Aires. LEMIT. Serie II. N ${ }^{\circ}$ 33. La Plata, 1950.

FRENGUELLI, J. Loess y Limos Pampeanos. Facultad de Ciencias Naturales y Museo de La Plata. Serie Técnica y Didáctica. № 7. La Plata, 1955.

IRIONDO, M. Climatic changes in the South American plains: Records of a continent-scale oscilation. Quaternary International. 57-58: 93-112, 1999.

JUÁREZ BADILLO, E. y RICO RODRíGUEZ, A. Mecánica de Suelos. Editorial Limusa, 2da. Edición. Madrid. 387 p., 1982.

LAMBE, W. y WHITMANN, R. Mecánica de Suelos. Editorial Limusa. Madrid. 436 p., 1976.

MAURO, M., PELLEGRINO, A, RAMONDINI, M. y URCIULI, G. A Contribution to the geotechnical characterization of large areas for landing planning. pp. 195-201. En: Geotechnical Site Characterization. Robertson \& Moyano (eds.). Editorial Balkema. Rotterdam, 1998.

PEA EI. 1.1. INSTITUTO de SUELOS. INTA (Coord.). Universidad Nacional de Jujuy, Universidad Nacional de Salta, EEA CerrillosINTA. Cartografía Temática Digital del Territorio Argentino de la Alta Cuenca del Río Bermejo; (mapa base, geología, geomorfología, hidrología, suelos, vegetación, población e infraestructura, uso de suelos). Instituto Nacional de Tecnología Agropecuaria. Buenos Aires. Informe inédito, 1999a.

PEA El. 2.5. ADMINISTRACIÓN PROVINCIAL del AGUA (Coord.), Centro de Geociencias Aplicadas (UNNE), Departamento de 
Hidraúlica (UNNE), INTA - E.E.R.A. Saénz Peña, INTA - E.E.R.A. C. Benítez, Dirección de Suelos y Agua Rural de la Subsecretaría de Recursos Naturales y Medio Ambiente de la Pcia. del Chaco. Cartas Temáticas Digitalizadas de la Cuenca Inferior del Río Bermejo. Elemento 2.5: Uso de la Tierra en la Cuenca del Río Bermejo. Chaco. Informe inédito, 1999b.

PEA El. 1.1. BREA, D. Generación y Transporte de Sedimentos en la Alta Cuenca del Río Bermejo. Impacto en la Hidrovía, Delta del Paraná y Río de la Plata. Instituto Nacional del Agua y del Ambiente. Elemento 1.1.: Movimiento Transfronterizo de Contaminantes. Buenos Aires. Informe inédito, 1999c.

PEA El. 2.5. ARRIETA, J.; PASTOR, C. Relevamiento Socioeconómico y Ambiental de las Comunidades del Tramo Medio e Inferior de la Cuenca del Río Bermejo. Informe y Anexos. Elemento: 2.5: Uso del Suelo en la Cuenca del Río Inferior. Buenos Aires. Informe inédito, 1998c.

PEA El. 2.6. CARDOZO, J. Manejo del Estrato Forrajero Mediante el Uso del Agua en Esteros, Bañados y Cañadas y Recuperación Productiva de Tierras Invadidas por Vinal. Elemento 2.6: Manejo de Forrajes. Chaco Húmedo. Chaco. Informe inédito, 1999d.

PEA El. 6.1. LAURELLI E; VAGHI, A. La Cuenca del Río Bermejo en el Contexto Regional Elemento 6.1: Formulación del Programa Estratégico de Acción. Buenos Aires. Informe inédito, 1999e.

PEA El. 6.1. Diagnóstico Ambiental de la Cuenca del Río Bermejo. El. 6.1 Formulación del Programa Estratégico de Acción Ambiental. Buenos Aires. Informe inédito, $1999 f$.

PYE, K. The nature, origin and accumulation of loess. Quaternary Science Review. 14: 653-667, 1995.

SAYAGO, J. M. Aproximación regional al loess subtropical Argentino. Actas. I Congreso Argentino de Cuaternario y Geomorfología. La Pampa. pp 159-175, 1999.

TERZAGHI, K. y PECK, R. Mecánica de Suelos en la Ingeniería Práctica. Editorial El Ateneo. $4^{\text {ta }}$ Relmpresión. Buenos Aires. 722 p., 1980.

TORRA, R. Algunas manifestaciones de la litología en las formas del relieve del área Resistencia-Corrientes y sus alrededores. Tesina inédita. Departamento de Geografía. Facultad de Humanidades. Universidad Nacional del Nordeste. Resistencia 103 p., 1994.

TORRA, R. Genesis and Age of the Ituzaingó Formation sands, northeastern Argentina: a preliminary note. 18th Regional European Meeting of Sedimentology. GAEA Heidelbergensis Abstracts. Heidelberg. p. 340, 1997. 
TORRA, R. Estructuras sedimentarias marinas diagnósticas en las arenas de la Formación Ituzaingó (Mioceno medio), entre Itatí y Empedrado, Provincia de Corrientes, Mesopotamia de Argentina. Revista del Instituto de Geología y Minería. 12 (1): 75-86, 1998 .

TORRA, R. A Brief Stratigraphy and Paleogeography of the Miocene Sea at the Mesopotamia Region, Northeastern Argentina, South America. Geocongress 98. Geological Society of South Africa. pp. 79-82. Pretoria, 1998b.

TORRA, R. Detección de Antiguos Cauces del Río Paraná mediante el empleo de Imágenes Satelitales. III Reunión de Comunicaciones Científicas y Tecnológicas. Universidad Nacional del Nordeste. Actas. 4: 181-184. Resistencia, 1998c.

TORRA, R. El Estudio de los Sedimentos del Cuaternario de la Provincia del Chaco y Regiones Aledañas. III Reunión de Comunicaciones Científicas y Tecnológicas. Universidad Nacional del Nordeste. Actas. 4: 197-200. Resistencia, 1998d.

TORRA, R. Geología del subsuelo del Gran Resistencia y Regiones aledañas. Comunicaciones Científicas y Tecnológicas 1999. Tomo VII Ciencias Tecnológicas. pp. 169-172. Corrientes, 1999.

TORRA, R. Geochemistry of a Tidal Transgressive Heterolithic Succession: The Ituzaingó Formation (Middle Miocene), Argentina. Chinese Journal of Geochemistry. 19 (1): 52-57, 2000.

TORRA, R. El conurbano del Gran Resistencia: características geológicas y geotecnológicas del subsuelo. Provincia del Chaco, Argentina. Revista Arandú. Facultad de Ingeniería. Universidad Nacional del Nordeste. 3: 11-17, 2001.

TORRA, R. Sedimentologic evolution of the extensional Cenozoic Argentine basin based on siliciclastic littoral shallow marine deposits: an approach. International Congress SEDIMENT 2002. Frankfurt, 2002a.

TORRA, R. The origin of the "Pampeana" Formation (Pleistocene-Holocene) at the Argentina plains and surrounding areas: an approach. International Geological Correlation Program. Special Abstract Volume. Project No. 464. 2nd Annual Conference (South American Venue). San Pablo. pp. 77-82, 2002b.

TORRA, R. y MIÑO, F. D. Propiedades Mecánicas de las Pelitas de la Formación Ituzaingó (Mioceno medio), en la localidad de Empedrado, Provincia de Corrientes, Argentina. Comunicaciones Científicas y Tecnológicas 1999. Tomo VII Ciencias Tecnológicas. pp. 173-176. Corrientes, 1999. 


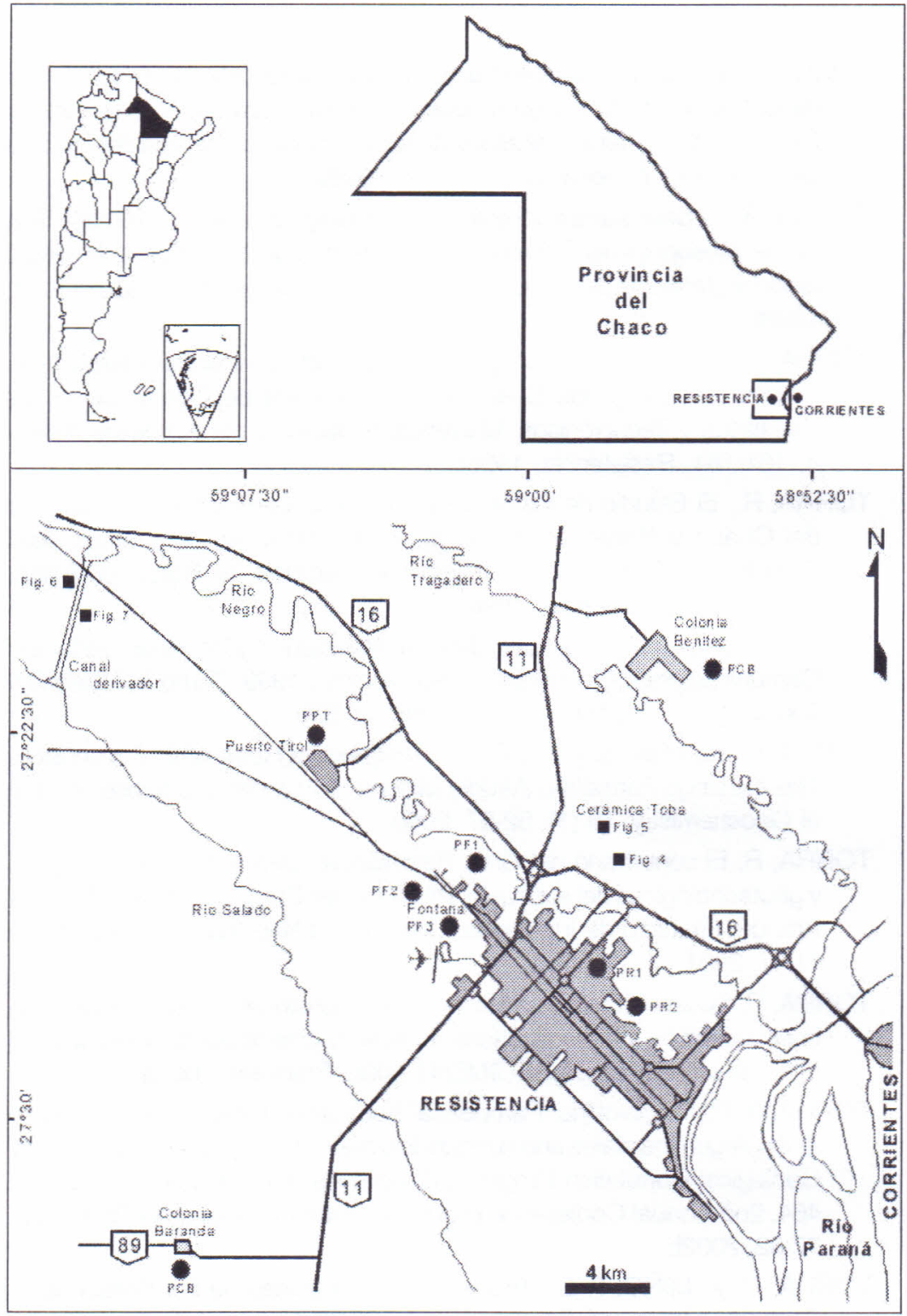

Fig. 1: Ubicación del área de estudio. PCB: Perforación Colonia Benítez; PR1-2: Perforaciones Resistencia 1 y 2; PF1-2-3: Perforaciones Fontana; PPT: Perforación Puerto Tirol; PCB: Perforación Colonia Baranda 


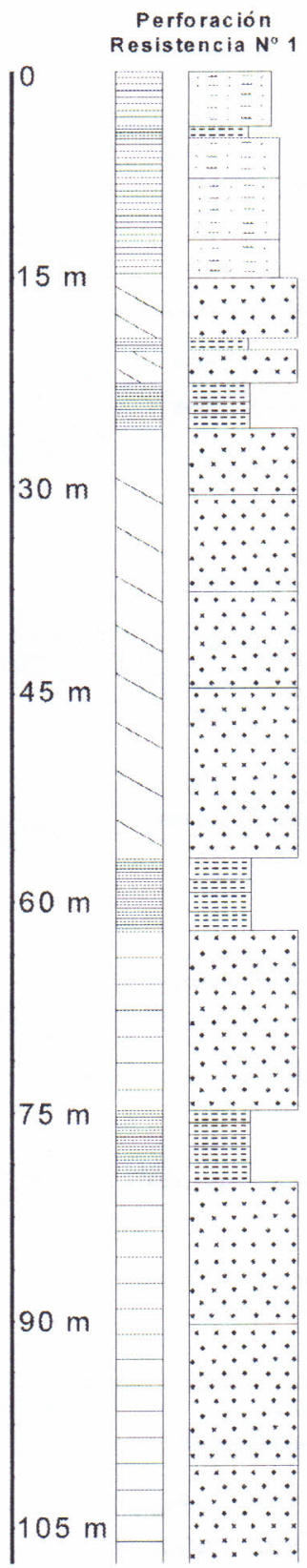

Perforación
Resistencia $2^{\circ}$
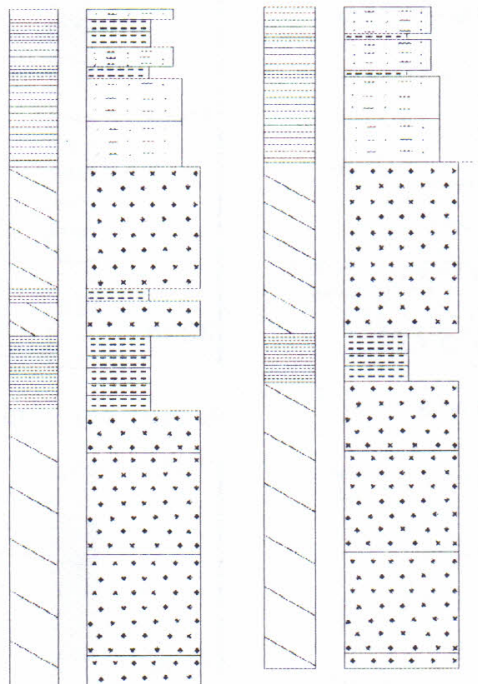

Formación Ituzaingó (Mioceno. Plioceno)

\section{REFERENCIAS}

\section{Textura}

$\therefore=\ldots$ limos fluviales y $(0)$ limos loésicos edafizados

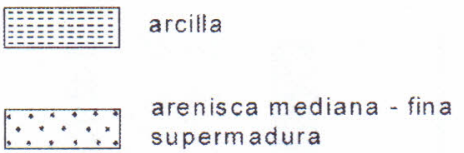

\section{Color}

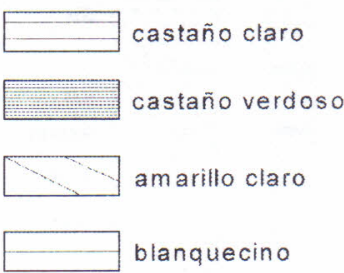

Autor: Roberto Torra, (2001) Figura 2

Fig. 2: Perforación Resistencia 1,2; Perforación Escuela Benítez 
Perforación Fontana $N^{0} 1$

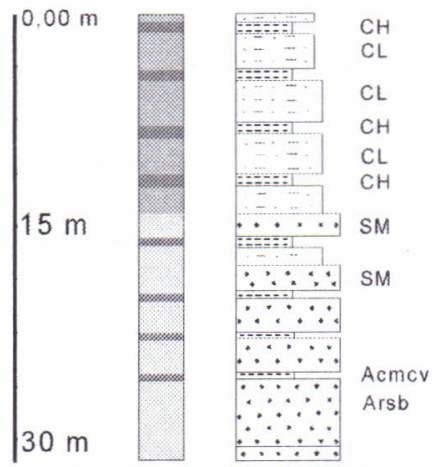

Perforación Fontana $\mathrm{N}^{\circ} 3$
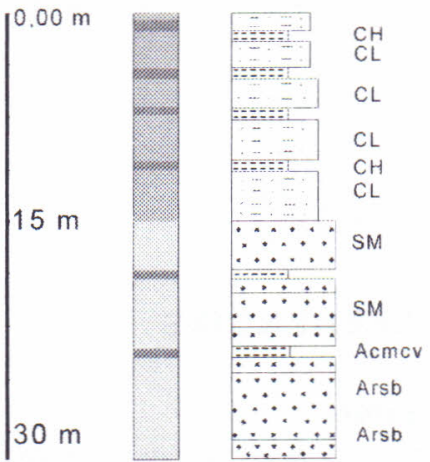

Perforación Colonia Baranda

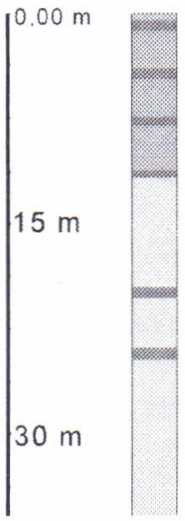

SM

AcmcV

$\because \because \because \therefore$ Arsb

$\therefore \therefore \therefore \therefore$

$\because \because \therefore \therefore$
Perforación Fontana $\mathrm{N}^{\circ} 2$

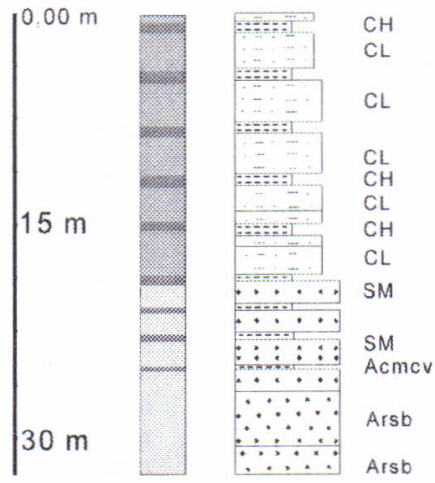

\section{Perforación Pto. Tirol}

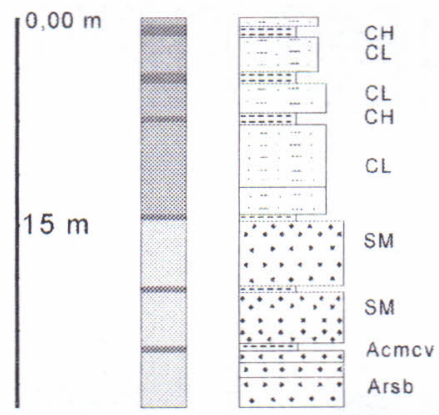

\section{REFERENCIAS}

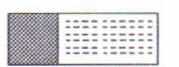

Arcilla de alta plasticidad (CH)

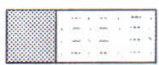

Arcilla de plasticidad media (CL)

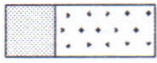

Arena fina limosa (SM)

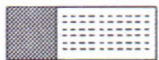

Arcilla maciza castaño verdosa ( $\mathrm{Acmcv}$ )

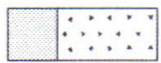

Arenisca fin a supermadura muy densa

blanquecina (Arsb)

Autor: Roberto Torra, (2001)
Figura 3

Fig. 3: Perforaciones Fontana 1,2,3; Perforación Puerto Tirol; Perforación Colonia Baranda 


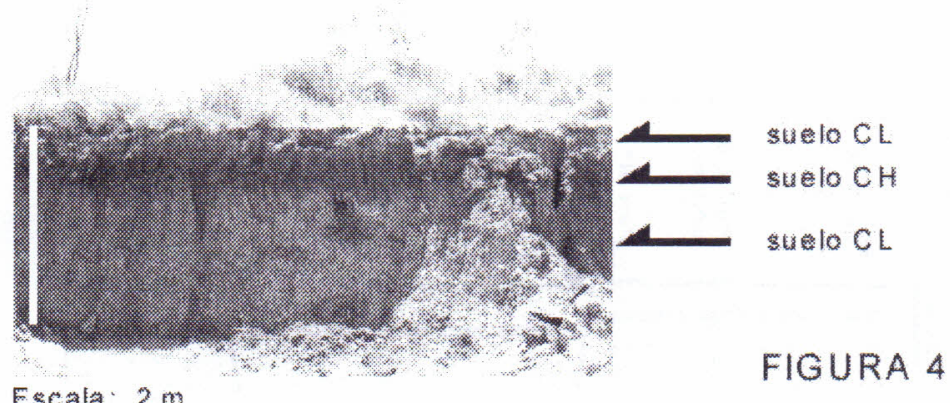

Escala: $2 \mathrm{~m}$

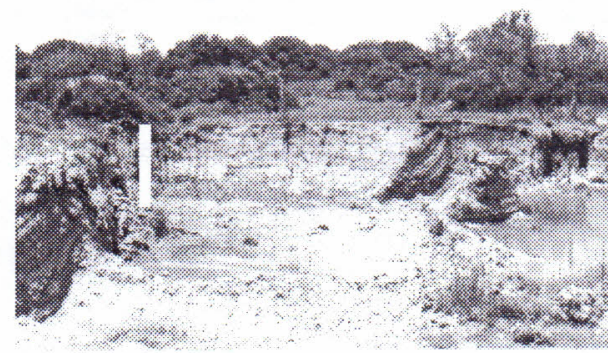

Escala: $2 \mathrm{~m}$

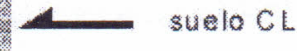

\section{FIGURA 5}

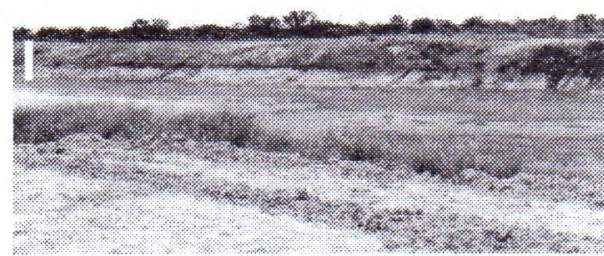

Escala: $6 \mathrm{~m}$

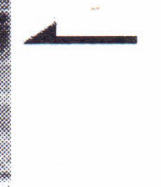

vista panoramica tallad

canal derivator

FIGURA 6
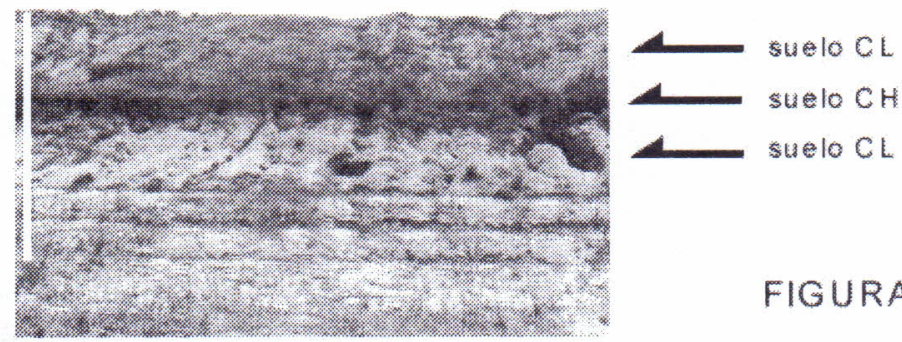

Escala: $6 \mathrm{~m}$

Autof: Roberto Tora, (2001)

Fig. 4, 5, 6, 7: Fotografias de los lugares de muestreo. Figs. 4, 5: destapes artificiales en las cercanías de la Cerámica Toba; Figs. 6 , 7: vistas del canal derivador del Río Negro 


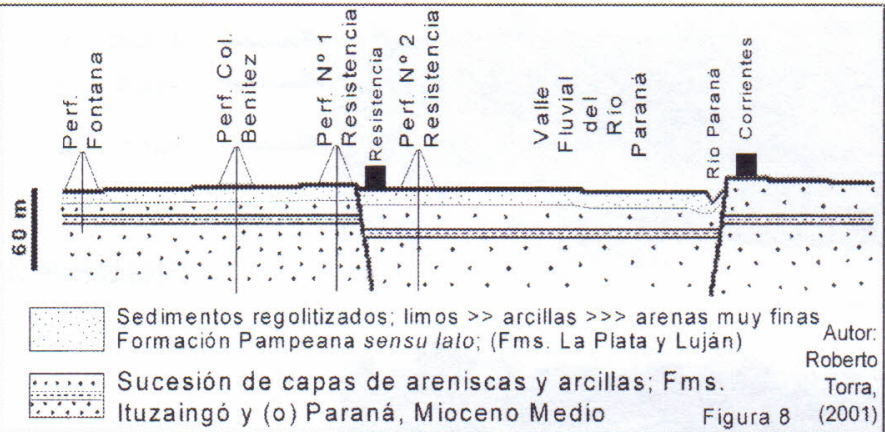

Fig 8: Perfil transversal al valle fluvial de Río Paraná a la altura del AMGR

RoBERTO TORRA

roberto_torra@arnet.com.ar

Departamento de Geociencias -Centro de Geociências Aplicadas

Facultad de Ingeniería - Universidad Nacional del Nordeste Avda Las Heras 727, H3500COI, Resistencia, Chaco

Argentina 